\title{
Good Relations between Nurses and Patients: Spreading the Wings of Imagination, Protecting Patients' Souls
}

\author{
Mayumi Uno $^{1 *}$, Yukari Katayama² \\ ${ }^{1}$ Department of Nursing Science, Graduate School of Medicine, Osaka University, Osaka, Japan \\ ${ }^{2}$ Faculty of Nursing, Doshisha Women's College of Liberal Arts, Kyoto, Japan \\ Email: *unomayu@gmail.com
}

How to cite this paper: Uno, M. and Katayama, Y. (2018) Good Relations between Nurses and Patients: Spreading the Wings of Imagination, Protecting Patients' Souls. Open Journal of Nursing, 8, 248-256. https://doi.org/10.4236/ojn.2018.84021

Received: March 21, 2018

Accepted: April 23, 2018

Published: April 26, 2018

Copyright ( 92018 by authors and Scientific Research Publishing Inc. This work is licensed under the Creative Commons Attribution International License (CC BY 4.0).

http://creativecommons.org/licenses/by/4.0/ (c) (i) Open Access

\begin{abstract}
Objective: To ascertain a method for building good nurse-patient relations from the narratives of veteran nurses who have accumulated profound nursing experience, and to show these phenomena in model form. Methods: Semi-structured interviews were conducted, verbatim records of the contents of the interviews produced, and qualitative analysis undertaken. Results of analysis and concepts considered important were shown in model form. Results: Veteran nurses, utilizing their nursing specialization, showed "Concern as humans," transcending their professional specialty. This was expressed as "spreading the wings of imagination" in order to know what is important to patients, and "protecting souls" in order to defend, together with patients, what is most important to the latter. "Spreading the wings of imagination to protect patients" souls" became the key to build good nurse-patient relations. Conclusion: Nurses with deep experience expressed building good nurse-patient relations as "spreading the wings of imagination and protecting patients' souls." We were able to ascertain that in nursing practice, using the sensitivity of veteran nurses, is one way of manifesting nursing conduct.
\end{abstract}

\section{Keywords}

Nurse-Patient Relations, Veteran Nurses, Nursing Practice, Sensitivity, Interview

\section{Research Background and Significance}

\subsection{Nursing Service Perspective}

Patient satisfaction in facilities such as hospitals is related to the quality of nurs- 
ing care and should be emphasized [1] [2].

It is internationally recognized that enhancement of core nursing services directly reflected in patients' fees, such as technology and life guidance, is required. In Japanese culture, it is required that the feelings of others be inferred without clear linguistic expression. In this way, entering into patients' subtle emotions, a sub-service not directly reflected in patients' fees, exerts an influence on patient satisfaction [3]. The sustainability of patient satisfaction is essential to the continuation of medical institutions. When patients are dissatisfied, they will not recommend those institutions to family, friends, and colleagues [4] [5] [6] [7]. Nurses bear a central role in healthcare teams in influencing patient satisfaction [8] and are part of a comprehensive medical system, while processes and structural factors affect the degree of patient satisfaction regarding nursing care [9]-[16].

Against the background of sophistication of medical devices, complexities of nursing duties, multitasking, etc., new nurses, to say nothing of veterans, have to do their utmost to steadily accomplish their duties, with a tendency to forget about violating patients' delicate feelings. In this case, a low evaluation will be accorded to patient-aware nursing services [17].

\subsection{Interview with a Nurse with Deep Experience}

Conflict between nurse and patient is cited as one scenario in which nurse-patient relations are unsatisfactory [3] [18] [19] [20]. Regarding conflict situations, a veteran nurse's response is flexible, and she is able to consider pre-emptive measures to prevent the conflict [21]. The researchers situated a "veteran" as not only defined by years of clinical experience, but also one deepening her own nursing practice through various relations with patients and renewing that experience in subsequent, enriched nursing practice. This paper suggests a veteran nurse to be a nurse with profound experience.

\subsection{Purpose}

The objective of this paper is to ascertain a method for building good nurse-patient relations from the narratives of veteran nurses and to show these phenomena in model form.

\section{Methods}

\subsection{Participant: A Designated Veteran Nurse}

The subject of this paper is an individual registered as a volunteer with a professional nursing association based in Osaka Prefecture, Japan. Volunteers are known as Gold Nurses and, having acquired full experience as nursing administratorsin healthcare facilities, following mandatory retirement, they participate in further healthcare activities.

In many regions of Japan, there are nursing professionals who register, after retirement, with professional nursing associations and participate in volunteer 
activities. The designation Gold Nurses is an original concept of Osaka Prefecture. The origin of the name signifies "Contributing to a sparkling society, even after retirement."

\subsection{Participants}

Among Gold Nurses, we focused on one veteran nurse from whom we had obtained agreement to participate.

She is a 70-year-old woman who graduated from a nursing school and was a head nurse.

\subsection{Data Collection}

In May 2015, we conducted an interview with the Gold Nurse concerning a specific mode of involvement that satisfactorily preserves nurse-patient relations. For the interview, a quiet room in the professional nursing association's building was prepared, with the researchers as interviewers, and an ambience of "Talking to Gold Nurses" created. An approximately 50-minute session, featuring open discussion of the theme was conducted. The interview contents, with the subject's permission, were captured on an IC recorder.

\subsection{Ethical Considerations}

The objective and methods of the research were explained verbally and in written form to the subject, and her consent was obtained. In addition, it was guaranteed that consent could be withdrawn at any time and that cooperation with the researchers would in no way be related to evaluation from the professional association where the subject was registered. The subject's approval of an ethical review of the researchers' previous affiliations was also obtained.

\subsection{Analysis Process}

The entire narrative obtained was set down in verbatim records. With the focus laid on the veteran nurse's mode of involvement with patients, qualitative, inductive analysis was performed, and the results displayed in a modeling diagram.

\section{Results}

\subsection{Excerpt from the Narrative}

\section{(Interviewer)}

How many years' experience have you had as a nursing professional?

(Veteran nurse)

After being a clinical nurse, I had administrative experience. Altogether, it comes to about 40 years.

(Interviewer)

In your nursing practice, what were your thoughts, and what was important to you in your dealings with patients?

(Veteran nurse) 
Well now, uninterrupted care from the acute to the recovery phase, up to the patient returning to the life they had before, means nursing involvement does not stop at supporting life, but is about protecting the soul of the patient at every stage. That's what I think nurses can be proud of.

We once had a Sashimonoshi admitted [Sashimonoshi: a Japanese cabinetmaker who creates craftwork, such as furniture, fittings, and personal effects by assembling wooden components without the use of nails or other joining equipment]. That gentleman had produced many artefacts with his hands. So I never inserted the intravenous line into the arm, his right arm, which did most of the work. It wasn't that he would be unable to use his right arm if we inserted an intravenous line there. I identified with that gentleman as to what was important to him. We were in the same boat, so to speak... If you put yourself in the patient's place, it's not a question of who is superior, who inferior, but trying to know that person to the very core. So, rather than a nursing specialist, what is fundamental is that $\mathrm{I}$, as a person, respect the other, regard the other as important. That's to say, it's about protecting the soul.

(Interviewer)

Is the source of "protecting the soul" connection to others as a person? Establishing deep connections as you, Ms. A [the veteran nurse], have done, does not seem to be the younger generation's strong point. That's to say, contact with recent nursing students revealed a very thorough protection of personal information, both on their own part and that of their counterparts, in their daily interactions. In other words, they seem not to have deep connections with others. That being the case, it would appear that, with regard to patients, as in human relations in their daily lives, new nurses believe that knowing more than necessary about others is undesirable. However, where is the line drawn for "necessary" in "more than necessary"? For example, while information needed in nursing training may be easy to ask for, in terms of an assessment framework for abstracting illness, treatment policy, and nursing issues, when it comes to detailed information and the patient's thoughts and feelings, it seems their perception is that one shouldn't ask. It seems that inquiring about these things during communication with patients is their weak point.

How do you think, then, one should be able to be aware of patients' thoughts and feelings?

(Veteran nurse)

Yes, it's a question of noticing thoughts and feelings, isn't it? I think sensitivity is needed for that. I believe it's possible to raise sensitivity through training. Previously, when I gave lectures at a nursing college, at the beginning of the lessons, I would show the students an object like a ballpoint pen or something and tell them that think for 3 minutes about things that you can see about this ballpoint pen, and find 5 things to say about it. At first, the students struggled to come up with something. However, on repeating the exercise, various viewpoints emerged, so this became training in viewing a single object and phenomenon 
from multiple angles.

I don't think sensitivity training can be implanted by reading books. Even if you read about something delicious, you won't know what it's like unless you try eating it. If you perceive things in a multifaceted way on a daily basis, you will, all of a sudden, be able to form various concepts.

Recently, visualization has been emphasized, and while this is a good thing, in a certain sense, I think it's trivial. So, while "spreading the wings of imagination" is all very well, without sensitivity, you won't be able to imagine or think about things. I think if this sensitivity is there, you can solve, together with the patient, the problems they have. I believe if that isn't the case, then you are not a nurse.

And then, while it's fine to imagine various things, making a unilateral decision is no good. Acting on the basis of confirmation with the patient is important.

(Interviewer)

Ah, I agree. Visualizing nursing, that is, verbalizing the phenomenon of nursing, is extremely important, and is our duty, I think. However, what you are saying, to make a comparison, is that sensitivity is like the lines between words, and not the words themselves. It is imagining unspoken thoughts and feelings, isn't it?

(Veteran nurse)

Yes, that's it. I have practiced nursing with that as a priority. Even if this wasn't true in all cases, I have experienced patients and their families opening their hearts and placing their trust in me.

(Interviewer)

Thank you. Ms. A's (the veteran nurse's) deep involvement is one method of preserving better connections with patients, isn't it? We have experienced something deep about human existence.

\subsection{Modeling of a Phenomenon Grasped from Narrative}

From this interview we learned that veteran nurses, using their nursing expertise, realized "human connections" that transcended nursing specialization. This was expressed as "spreading the wings of imagination," in order to know what is important to patients, and "protecting souls" so as to guard, together with patients, what is most important to them. "Spreading the wings of imagination" and "protecting patients' souls" were the key to building good nurse-patient relations. Building good relations, based on mutual trust between nurses and patients, is illustrated (Figure 1).

\section{Discussion}

\subsection{Universality of Nurse-Patient Relations}

From our interview with the veteran nurse, we ascertained a specific mode of involvement necessary for the satisfactory preservation of nurse-patient relations. The mode expressed as "spreading the wings of imagination" and "protecting 


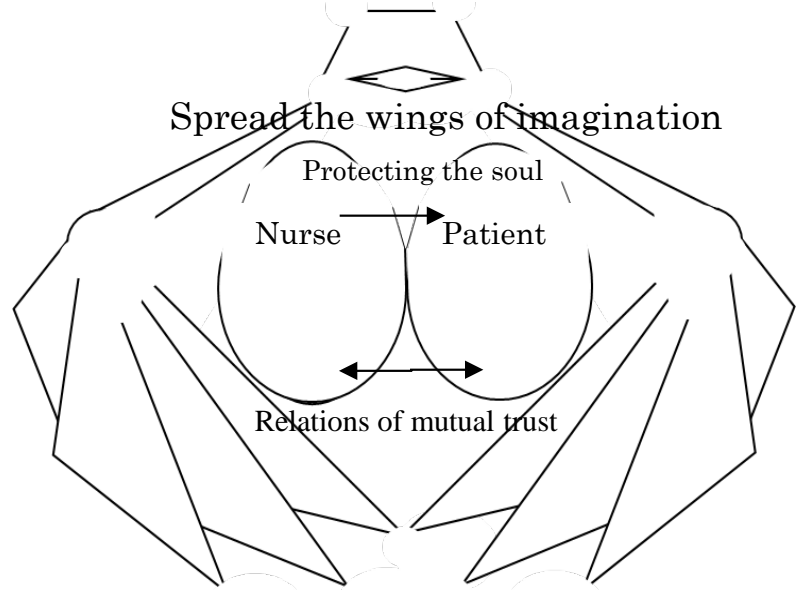

Figure 1. Veteran nurse's model of construction of good relations with patients.

patients' souls" can be described as both the concept in Japanese culture of "inferring the other's feelings" and "drawing close to the patient's feelings" [3]. The sense of wanting to be understood without conveying this verbally to the other party is referred to as "amae," a concept unique to Japanese culture. There is no English expression equivalent to "amae," which is the vague expression of feelings instinctively sensed [22].

On the other hand, Henderson proposed verbalization of the source of nurse-patient relations as "the ability to enter into his or her (the patient's) own skin" [23] and emphasized close observation of the patient's true wishes and consideration of the necessary nursing. Florence Nightingale, prior to generalization of the expression "sympathy" in nurse-patient relations, used this term in scientifically explaining a method for drawing close to the patient [24].

In light of the above, we were able to confirm from this research that the nucleus of building good nurse-patient relations, despite differences in linguistic expression due to cultural background, is universal.

\subsection{Conflict Due to Failure to Establish Mutual Trust}

Robbins defined conflict as a process originating in the point where it is recognized that another has exerted, or is attempting to exert, a negative influence on matters important to itself, explaining it not as a problem related to conflict-like emotions in interpersonal relations, but as the co-occurrence of changed emotions in both parties [25].

There are cases where, due to a temporary, emotional change on the part of the nurse or patient, relations between both parties deteriorate. Given the stressful circumstances under which the nurse has to master multitasking, the leeway in which to draw close to the patient may be lost. The patient is also subject to stressful circumstances, spending hours lying in bed, without stimulation and with unease about the future. In these ways, the nurse and the patient experience unsettling emotions due to differing stances. Where a disturbance of the 
patient's own delicate emotions cannot be conveyed to the nurse, or the nurse does not adopt an appropriate response to the patient's unsettled emotions, dissatisfaction will result. These circumstances lead to conflict between nurse and patient, and the patient suffers [20]. Suffering consists of feelings and emotional response in order to live, which shape the experience of a person living with illness, disability, or lifestyle problems [26] [27]. To sum up, it is conceivable that, where nurse-patient trust cannot be built, conflict easily occurs, due to some type of emotional unsettling, resulting in the patient's suffering.

\subsection{Nursing Practice Using Veteran Nurses' Sensitivity}

Even when the period of connection with the patient was short, the veteran nurse was able to build relations of trust. Generally, nurses within the veteran sphere possess sufficient nursing techniques.

However, the veteran nurse, who was the subject of this research, went beyond nursing related to treatment of diseases, and also tried to sense emotionally what was important to the patient in their lives, to know their personal history, and to consider their future. The veteran nurse expressed this as the "wings of imagination." "Imagination" does not suggest a range of thought based on scientific grounding such as conjecture but is considered as sensibility overflowing with humanity. In addition, the emotions geared to protecting the patient's thinking in common with the patient were expressed as "protecting souls." The patient's soul is the nucleus of their body, spirit, and sociability, and can be thought of as unifier of these elements.

\section{Conclusion}

Nurses with deep experience, as a foundation for building good nurse-patient relations, expressed "spreading the wings of imagination" as a means of knowing what is important to the patient and "protecting souls" as a means of protecting, together with the patient, what is most important to the latter. The expression "spreading the wings of imagination and protecting patients' souls" can also be said to be the concept, unique to Japanese culture, "inferring the other's feelings." The building by veteran nurses of good nurse-patient relations depends not only on the length of clinical experience, but also on a deepening of nursing practice. The result can be said to be that building good nurse-patient relations is enabled. In this way, nursing practice that uses sensitivity can be understood as one manifestation of nursing conduct.

\section{Limitations}

This study is the pursuit of a single story. There is a need to consider from multiple opinions.

\section{Acknowledgements}

Sincere thanks are extended to the veteran nurse who willingly participated in 
the interview.

\section{Conflict of Interest}

None.

\section{References}

[1] Otani, K., Kurz, R.S. and Barney, S.M. (2004) The Impact of Nursing Care and Other Healthcare Attributes on Hospitalized Patient Satisfaction and Behavioural Intentions. Journal of Healthcare Management, 49, 181-196. https://doi.org/10.1097/00115514-200405000-00008

[2] Sharma, S.K. and Kamra, P.K. (2013) Patient Satisfaction with Nursing Care in Public and Private Hospitals. Nursing and Midwifery Research Journal, 9, 130-141.

[3] Uno, M., Tsujimoto, T. and Inoue, T. (2014) Effect on Patients of Conflict-Generating Scenarios between Patients and Nurses. Journal of the Department of Nursing Science, 20, 47-53.

[4] Ellis-Jacobs, K.A. (2011) A Quantitative Correlational Study on the Impact of Patient Satisfaction on a Rural Hospital. Internet Journal Allied Health Sciences and Practice, 9, 1-6. http://ijahsp.nova.edu/articles/Vol9Num4/pdf/ellis-jacobs.pdf

[5] Lee, M.A. and Yom, Y.H. (2007) A Comparative Study of Patients' and Nurses' Perceptions of the Quality of Nursing Services, Satisfaction and Intent to Revisit the Hospital: A Questionnaire Survey. International Journal of Nursing Studies, 44, 545-555. https://doi.org/10.1016/j.ijnurstu.2006.03.006

[6] Shirley, E.D. and Sanders, J.O. (2013) Patient Satisfaction: Implications and Predictors of Success. Journal of Bone \&Joint Surgery, 95, e69.

[7] Ting, C.Y. and Yu, T.K. (2010) Modeling Patient Perceptions of Service Recovery: The Effects of Perceived Fairness on Health Center Repatronage. Social Behavior and Personality, 38, 395-403. https://doi.org/10.2224/sbp.2010.38.3.395

[8] Dzomeku, V.M., Ba-Etilayoo, A., Perekuu, T. and Mantey, R.E. (2013) In-Patient Satisfaction with Nursing Care: A Case Study at Kwame Nkrumah University of Science and Technology Hospital. International Journal of Research in Medical and Health Sciences, 2, 19-24.

[9] Donabedian, A. (1992) The Role of Outcomes in Quality Assessment and Assurance. Quality Review Bulletin, 18, 356-360. https://doi.org/10.1016/S0097-5990(16)30560-7

[10] Nelson, E.C., Mohr, J.J., Batalden, P.B. and Plume, S.K. (1996) Improving Health Care, Part 1: The Clinical Value Compass. Joint Commission Journal on Quality Improvement, 22, 243-258. https://doi.org/10.1016/S1070-3241(16)30228-0

[11] Irvine, D., Sidani, S. and Mc Gillis, L.H. (1998) Linking Outcomes to Nurses' Roles in Health Care. Nursing Economics, 16, 58-64.

[12] Duffy, J.R. and Hoskins, L.M. (2003) The Quality-Caring Model: Blending Dual Paradigms. Advances in Nursing Science, 26, 77-78. https://doi.org/10.1097/00012272-200301000-00010

[13] Korniewicz, D.M. and Duffy, J. (2008) Essential Concepts for Staff Nurses: The Outcomes Imperative. American Nurses Association, Continuing Education, USA.

[14] Mark, B., Salyer, J. and Wan, T.T.H. (2003) Impact on Organizational and Patient Outcomes: Professional Nursing Practice. Journal of Nursing Administration, 33, 224-234. https://doi.org/10.1097/00005110-200304000-00008 
[15] Yen, M. (2004) A Model for Testing the Relationship of Nursing Care and Patient Outcomes. Nursing Economics, 22, 75-80.

[16] Tzeng, H.M. and Yin, C.Y. (2008) Patient Satisfaction versus Quality. Nursing Ethics, 15, 121-124. https://doi.org/10.1177/0969733007080210

[17] Uno, M., Tsujimoto, T. and Inoue, T. (2017) Features of Suffering Perceived by Japanese Patients in a Stable Condition: A Text Analysis. Open Journal of Nursing, 7, 1021-1033. https://doi.org/10.4236/ojn.2017.79074

[18] Uno, M., Tsujimoto, T. and Inoue, T. (2017) Perceptions of Patients' Expectations of Care among Nurses in Japan: A Qualitative Study. International Journal of Nursing Sciences, 4, 58-62. https://doi.org/10.1016/j.ijnss.2016.12.005

[19] Uno, M. and Tsujimoto, T. (2017) Characteristics of Nurse Perceptions Concerning Nurse-Patient Conflict: Analysis by Text Mining as a Complement to Qualitative Descriptive Analysis. Japan Journal of Human Health Care, 2, 1-12.

[20] Uno, M. (2018) The Concepts of "Patient Suffering" and "Favorable Interaction" in Nurse-Patient Conflicts Based on Gaps in Perception. Biomedical Journal of Scientific \& Technical Research, 2, 1-8. https://doi.org/10.26717/BJSTR.2018.02.000666

[21] Uno, M., Ikuta, S. and Okamoto, M. (2016) Aspects of Avoidance of Conflict between Nurses and Patients, According to Japanese Gold Nurses (or Expert Nurses): A Program for Raising the Quality of Nursing. Journal of Yamato University, 2, 91-97.

[22] Doi, T. (2010) Amae to Nihonjin. Kadokawa-Syuppan, Japan. (In Japanese)

[23] Henderson, V. (1964) The Nature of Nursing. American Journal of Nursing, 64, 62-68.

[24] Seymer, L. (1954) Selected Writings of Florence Nightingale. Macmillan Publishers, New York.

[25] Robbins, S.P. (2009) Organizational Behavior. 8th Edition, Diamond, Japan. (In Japanese)

[26] Kleinman, A. and Kleinman, J. (1995) Suffering and Its Professional Transformation: Toward an Ethnography of Interpersonal Experience. In: Kleinmen, A., Ed., Writing at the Margin: Discourse between Anthropology and Medicine, University of California Press, Berkeley, CA, 95-119.

[27] Travelbee, J. (1971) Interpersonal Aspects of Nursing. F. A. Davis, Philadelphia. 